\title{
Human STING Is Regulated by an Autoinhibitory Mechanism for Type I Interferon Production
}

\author{
Shishi Qia,b,c Changwan Wang ${ }^{b, c}$ Rui Zhang ${ }^{b, c}$ Junyan Zhub,c \\ Xianteng Hou ${ }^{b, c}$ Yingbo Jiang ${ }^{\text {b, c }}$ Jiaxin $\mathrm{Li}^{\mathrm{b}, \mathrm{c}}$ Miao Ren ${ }^{\mathrm{b}, \mathrm{c}}$ Muwang $\mathrm{Li}^{\mathrm{a}}$ \\ Fajian Hou ${ }^{b, c, d}$
}

ajiangsu Key Laboratory of Sericultural Biology and Biotechnology, School of Biotechnology, Jiangsu University of Science and Technology, Zhenjiang, China; ${ }^{b}$ State Key Laboratory of Molecular Biology, Shanghai Institute of Biochemistry and Cell Biology, Center for Excellence in Molecular Cell Science, Chinese Academy of Sciences, Shanghai, China; 'University of Chinese Academy of Sciences, Beijing, China; ${ }^{d}$ School of Life Science, Hangzhou Institute for Advanced Study, University of Chinese Academy of Sciences, Hangzhou, China

\section{Keywords}

Innate immune $\cdot$ Stimulator of interferon genes .

Interferon · Nuclear factor kappa-light-chain-enhancer of activated $\mathrm{B}$ cell $\cdot$ Interferon regulatory factor 3

\begin{abstract}
Stimulator of interferon genes (STING) plays a pivotal role in type I interferon-mediated innate immune response to the cytoplasmic detection of aberrant DNA. STING is a membrane protein localized in endoplasmic reticulum (ER), which upon stimulation translocates to Golgi apparatus and activates downstream signaling cascades. However, the mechanism regulating STING activity and significance of its intracellular traffic are not completely understood. Here we identify a novel region of human STING comprising thirteen residues within its C-terminal tail (CTT) for downstream nuclear factor kappa-light-chain-enhancer of activated $\mathrm{B}$ cell (NF-KB) activation. We also discover that STING $\mathrm{CTT}$ fragment can activate downstream signaling regardless of its ER localization. In addition, we reveal that ligand-
\end{abstract}

karger@karger.com www.karger.com/jin

Karger"

BOPEN ACCESS
(C) 2022 The Author(s)

Published by S. Karger AG, Basel

This is an Open Access article licensed under the Creative Common Attribution-NonCommercial-4.0 International License (CC BY-NC) (http://www.karger.com/Services/OpenAccessLicense), applicable to the online version of the article only. Usage and distribution for commercial purposes requires written permission. binding domain (LBD) in the middle of STING binds and confers autoinhibition to its CTT for both NF-KB- and interferon regulatory factor 3-activation. Furthermore, STING LBD can inhibit the interferon-stimulating activity of STING $\mathrm{CTT}$ in trans and demonstrate a dominant negative effect on endogenous STING for interferon induction. We thus uncover an important autoinhibitory mechanism modulating STING activity.

(c) 2022 The Author(s).

Published by S. Karger AG, Basel

\section{Introduction}

Viral infection elicits a potent innate immune response in host cells as an evolutionarily conserved defense mechanism $[1,2]$. Once in cells, viral nucleic acids can be taken as a foreign or danger signal and detected by pattern rec-

Shishi Qi, Changwan Wang, and Rui Zhang contributed equally to this work.
Correspondence to:

Fajian Hou, fhou@sibcb.ac.cn 
ognition receptors such as cyclic GMP-AMP synthase (cGAS) and RIG-I-like receptors [3-5]. cGAS senses the cytoplasmic presence of foreign or mislocalized self DNA and catalyzes the synthesis of dinucleotide $2^{\prime}-5^{\prime}, 3^{\prime}-5^{\prime} \mathrm{cy}$ clic GMP-AMP ( $\left(2^{\prime} 3^{\prime}\right.$-cGAMP), which binds to and causes stimulator of interferon genes (STING) activation [6-8]. The adaptor STING (also known as MITA, ERIS, MPYS, and TMEM173) then activates downstream signaling cascades, culminating in nuclear factor kappa-lightchain-enhancer of activated B cell (NF- $\mathrm{kB})$ and interferon regulatory factor 3 (IRF3) activation and their translocation into nucleus to turn on type I interferon (IFN-I) expression [9-11]. IFN-I executes its well-known antiviral function by inducing an antiviral cellular state as well as a variety of immunomodulatory effects in an autocrine or paracrine manner.

Human STING contains three major segments, i.e., four N-terminal transmembrane helices for its endoplasmic reticulum (ER) localization, a middle ligand-binding domain (LBD) for binding to cGAMP and a C-terminal tail (CTT) [12]. Binding to cGAMP triggers STING translocation from ER to Golgi apparatus though the ER-Golgi intermediate compartment (ERGIC) [13]. Only on arriving at the Golgi compartment can STING activate downstream signaling cascades for IFN-I induction, suggesting a very elegant mechanism regulating STING activation. Posttranslational modifications have been proposed to regulate STING activity [14-16]. A recent report showed that Golgi apparatus-synthesized sulfated glycosaminoglycans mediate STING activity [17]. Despite these studies, how the IFN-Inducing activity of STING is regulated precisely remains to be dissected. In addition to IFN-I induction, STING can also stimulate autophagy via ER-Golgi intermediate compartment and LC3 lipidation, which might be a primordial and conserved antiviral function and presumably predates its IFN-Inducing activity in evolution [18].

STING activates the downstream TBK1-IRF3 axis for IFN-I production though its CTT fragment. STING CTT contains a conserved pLxIS motif shared by a few adaptor proteins involved in innate immune signaling, which can signal through engineered myddosomes in a TLR signal-dependent manner [19-23]. pLxIS motif provides a docking site for IRF3, which is phosphorylated and thereby activated by TBK1. TBK1 is recruited to STING by a specific TBK1-binding motif in STING CTT, and a conserved PLPLRT/SD motif within STING CTT binds directly to the dimer interface of TBK1 [19]. Recent structural studies revealed that in the absence of infection, STING forms a butterfly-shaped dimer on ER and

Human STING Is Regulated by an

Autoinhibitory Mechanism its LBD adopts an inactive conformation. Binding to cGAMP triggers a $180^{\circ}$ rotation of LBD relative to the transmembrane domain (TM), followed by a conformational change leading to STING oligomerization and activation [12]. During the process, how STING CTT becomes active is not known, since it was invisible in the cryo-EM maps of apo STING. In contrast to the elaborated studies on the activation of TBK1-IRF3 axis by STING, much less is known on how NF- $\kappa \mathrm{B}$ axis is activated, which is required for sufficient IFN-I induction and as important as the TBK1-IRF3 axis. Several reports showed the NF- $\kappa \mathrm{B}$-stimulating activity of STING [2427]. A recent study revealed a unique CTT module of zebrafish STING, which results in pronounced NF- $\kappa B$ activation and is conserved in ray-finned fish [28]. This module is actually a CTT extension and constitutes a TRAF6-binding site, providing the mechanistic basis for its robust NF- $\kappa B$-stimulating activity. However, this zebra fish CTT extension is completely absent from human STING, raising an intriguing question on how human STING activates NF- $\kappa B$ axis to accomplish IFN-I induction together with TBK1-IRF3.

In this report, we sought to determine how specific region or elements of STING CTT are assigned to activate downstream signaling and reveal regulatory mechanisms involved. We found that STING CTT can activate not only IRF3 as previously reported but also NF- $\kappa \mathrm{B}$, and then identified a previously unknown region of STING CTT (aa-345-357) that can activate NF-kB axis. Subsequently, we revealed that STING CTT fragment can signal from mitochondria to stimulate IFN-I expression. Furthermore, we unexpectedly uncovered that STING LBD can bind to its CTT and modulate CTT signaling through an autoinhibitory mechanism.

\section{Materials and Methods}

\section{Plasmids}

Human complementary DNA was prepared from total RNA of HEK293 cells, and STING cDNA was subcloned into pcDNA3FLAG expression vector. Primers used for the amplification can be found in online supplementary Table S1 (for all online suppl. material, see www.karger.com/doi/10.1159/000521734). pcDNA3FLAG-SUMO-MAVS (aa-501-540) was described previously [29]. All mutations and deletions were constructed with the Fastmutagenesis Kit (Transgene Biotech) or overlapping PCR strategy, and primers are shown in online supplementary Table S2. All constructs were confirmed by DNA sequencing.

Cell Culture and Transfection

HEK293T cells, HEK293 cells, Vero cells, and TBK1-deficient HEK293T cells were cultured in DMEM medium (Hyclone) sup- 
plemented with 10\% FBS (ExCell Bio), $100 \mathrm{U} / \mathrm{mL}$ penicillin, and $100 \mu \mathrm{g} / \mathrm{mL}$ streptomycin (Gibco) [30, 31]. All cells were cultured at $37^{\circ} \mathrm{C}$ in a humidified incubator with $5 \% \mathrm{CO}_{2}$. HEK293 or HEK293T cells were seeded in 12 -well plates at a density of $1 \times 10^{5}$ cells per well and $5 \mathrm{~h}$ later, plasmids were transfected into cells using Lipofectamine 3000 (Thermo Fisher-Invitrogen). Cells were harvested for RT-qPCR assay, luciferase reporter assay, or Western blot analysis $24 \mathrm{~h}$ after transfection. Firefly luciferase assay and qPCR were performed after infection with HSV-1-GFP for $12 \mathrm{~h}$. For overexpression of various STING, $500 \mathrm{ng}$ of plasmids expressing STING or cGAS were transfected into cells as indicated, while for low expression of STING and cGAS, $50 \mathrm{ng}$ of plasmids were used.

\section{Antibodies}

Commercial antibodies included rabbit anti-FLAG (Santa Cruz), rabbit anti-HA (Santa Cruz), mouse anti-tubulin (Sigma), mouse anti-HA (Cell Signaling Technology), rabbit anti-cGAS (Cell Signaling Technology), and mouse anti-STING (Proteintech). Mouse anti-tubulin antibody was used for loading controls, and all mentioned antibodies were used at a dilution of 1:5,000 in $5 \%$ bovine serum albumin (BSA).

\section{Quantitative PCR}

Total RNA was extracted using TRIzol ${ }^{\circledR}$ reagent (Tiangen) from HEK293T cells, and cDNA was synthesized with HiScript ${ }^{\circledR}$ III RT SuperMix (Vazyme). Quantitative RT-PCR (qPCR) reactions and analysis were performed using Roche Lightcycler 96. ChamQ Universal SYBR qPCR Master Mix (Vazyme) was used for SYBR Green-based RT-qPCR according to the manufacturer's protocol. As internal controls, GAPDH expression was measured for HEK293T cells. Induction fold was determined by the $\Delta \Delta \mathrm{Cq}$ method and qPCR primers used to amplify specific genes are shown in online supplementary Table S1.

\section{Immunoprecipitations}

HEK293T cells were transfected with expression vectors as indicated. Forty-eight hours post transfection, cells were washed with cold PBS buffer and resuspended with lysis buffer (HEPES 20 $\mathrm{mM}$ [pH 7.5], $\mathrm{NaCl} 150 \mathrm{~mm}$, Triton X-100 0.5\%, TCEP $1 \mathrm{mM}$, and PMSF $1 \mathrm{mM}$ ). Cellularlysate wasincubated with Anti-DYKDDDDK affinity beads (Smart Lifesciences) at $4^{\circ} \mathrm{C}$ for $4 \mathrm{~h}$. The antiDYKDDDDK affinity beads were then collected and washed three times with lysis buffer. IP products were then subjected to SDSPAGE and immunoblotting.

\section{Western Blotting}

Cells were lysed with RIPA-150 buffer (Tris- $\mathrm{HCl} 50 \mathrm{mM}, \mathrm{pH}$ 7.4, NaCl 150 mM, Triton X-100 1\%, SDS 0.1\%, sodium deoxycholate $1 \%, \mathrm{NP}-401 \%$, and EDTA $0.5 \mathrm{~mm}$ ) supplemented with protease inhibitor cocktail (Roche). Protein concentrations were determined by Bradford reagent (Bio-Rad). Cell lysates were mixed with SDS-PAGE sample buffer and boiled for $5 \mathrm{~min}$ prior to SDSPAGE. Proteins in gel were transferred onto PVDF membranes (Millipore). After gel transfer and milk blocking, the membranes were incubated with primary antibody at room temperature for 1 h or $4^{\circ} \mathrm{C}$ overnight. After three washes with TBST $(1 \times$ TBS, $0.02 \%$ Tween), the membranes were incubated with secondary antibodies for $1 \mathrm{~h}$ at room temperature. After three washes with TBST, signals on the membrane were visualized by ECL (Share-Bio) and scanned by MiniChemi ${ }^{\mathrm{TM}}$ (Sagecreation), and the blots were analyzed by Sage Capture Software.

\section{Viral Infection}

Recombinant virus VSV-DM51-GFP and HSV-1-GFP was amplified in HEK293T cells and Vero cells, respectively, and used with a multiplicity of infection (MOI) of 1. For VSV-DM51-GFP or HSV-1-GFP infection, the cells were seeded in 12-well plates at a density of $1 \times 10^{5}$ cells per well. Virus was diluted at a certain ratio in serum free medium and then incubated with cells for $1 \mathrm{~h}$ in $\mathrm{CO}_{2}$ incubator, followed by addition of equal volume of fresh culture medium containing 10\% FBS. Cells infected with VSV-DM51GFP were harvested $8 \mathrm{~h}$ post infection for following analysis, and cells with HSV-1-GFP infection were harvested after $12 \mathrm{~h}$ for following analysis. VSV-DM51-GFP was described in our previous publication [30-32].

\section{Fluorescence Microscopy}

Using Olympus IX71 inverted fluorescence microscope, images were taken $8 \mathrm{~h}$ after VSV infection or $12 \mathrm{~h}$ post HSV infection.

\section{Luciferase Reporter Assay}

HEK293T cells were seeded in 12 -well plates at a density of $1 \times$ $10^{5}$ cells per well and $5 \mathrm{~h}$ later transfected with $20 \mathrm{ng}$ of reporter gene (IFN-I-luciferase, ISRE-luciferase or NF- $\kappa \mathrm{B}$-luciferase), 20 ng of pCMV-LacZ as internal control, and indicated expression vectors by Lipo 3000 (Thermo Fisher-Invitrogen). Twenty-four hours post transfection, cells were stimulated with or without virus. Cells were then harvested and lysed in Passive Lysis Buffer (Promega). Firefly luciferase activities were measured with a luminometer using the Luciferase Reporter Kit (Promega) and LacZ activities were measured by o-nitrophenyl-b-D-galactopyranoside (ONPG) assay in buffer $\left(\mathrm{Na}_{2} \mathrm{HPO}_{4} / \mathrm{NaH}_{2} \mathrm{PO}_{4} 200 \mathrm{mM}, \mathrm{pH}\right.$ 7.3, $\mathrm{MgCl}_{2} 2 \mathrm{mM}$, b-mercaptoethanol $100 \mathrm{mM}$, and ONPG $1.33 \mathrm{mg} /$ $\mathrm{mL}$ ) following a protocol provided by Sigma Technical Bulletin (GALA-1KT). Fold induction of firefly luciferase was normalized to LacZ activity. Data were shown as fold induction over empty vector-transfected controls.

\section{Viral Plaque Assay}

Plaque assay was performed as previously described [29]. In brief, HEK293T cells in 6-well plates were infected with VSVDM51-GFP or HSV-1-GFP for $1 \mathrm{~h}$. The infected cells were overlaid with $1 \%$ soft agar dissolved in DMEM and incubated for $48 \mathrm{~h}$. Plates were stained with $0.1 \%$ crystal violet in DMEM to display plaques, which were then quantitated.

\section{Statistics and Reproducibility}

All data are shown as mean values \pm SD based on data from three independent experiments. Statistics significance between two groups was determined by two-tailed Student's $t$ test, and a $p$ value of less than 0.05 was recognized as a statistically significance.

\section{Data Availability}

The data that support the findings of this study are available from the corresponding author on reasonable request. 
a

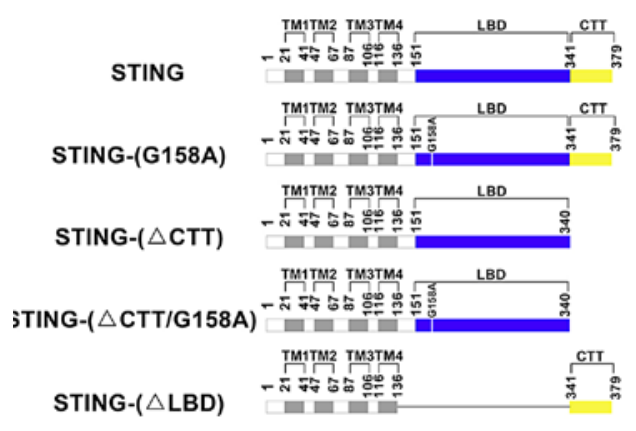

b

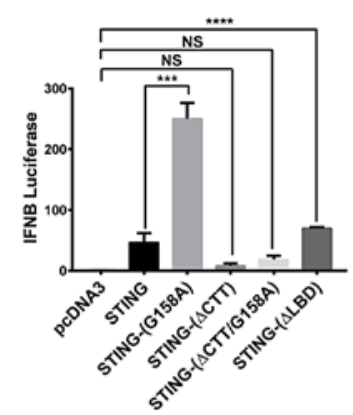

C

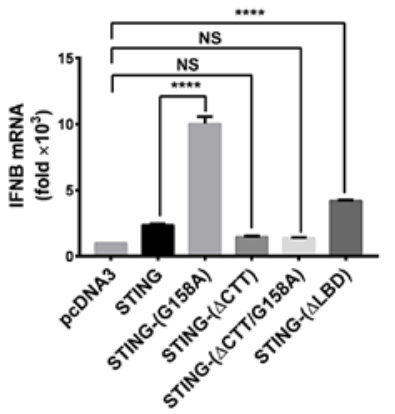

d

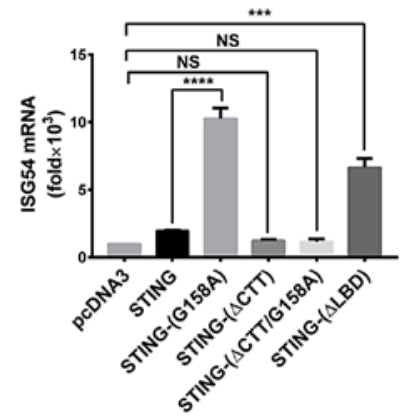

g
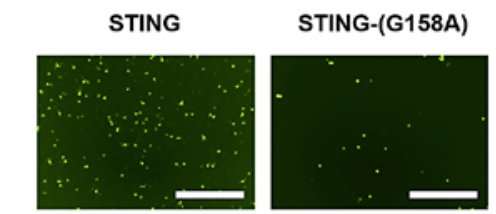

STING-( $\Delta C T T / G 158 A)$
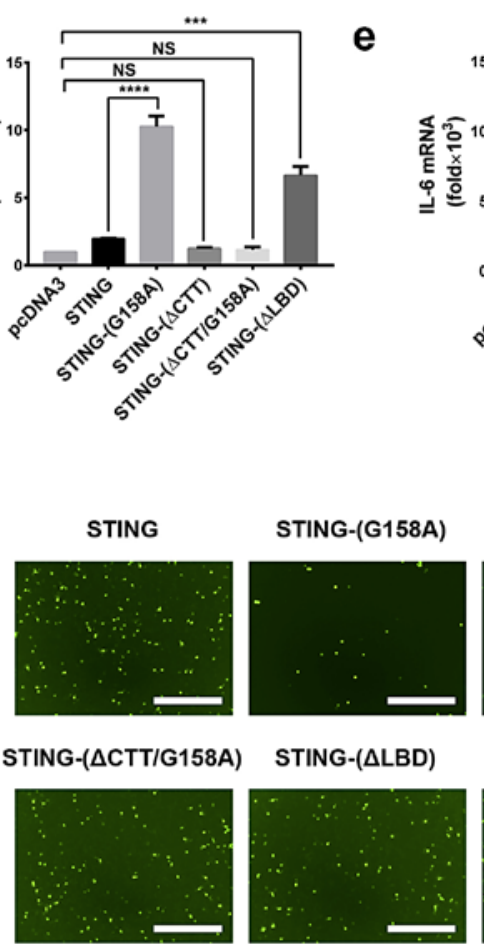
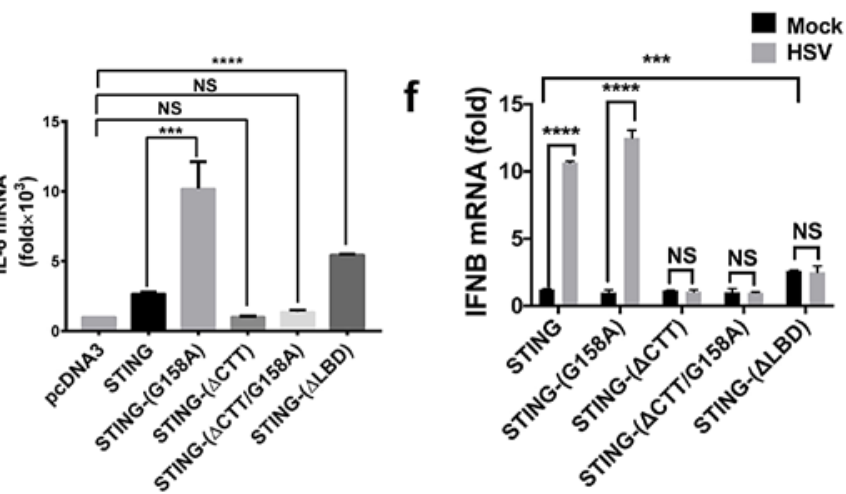

\section{STING-( $\Delta C T T)$}

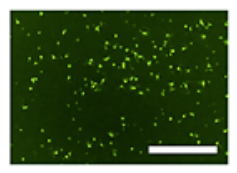

Mock
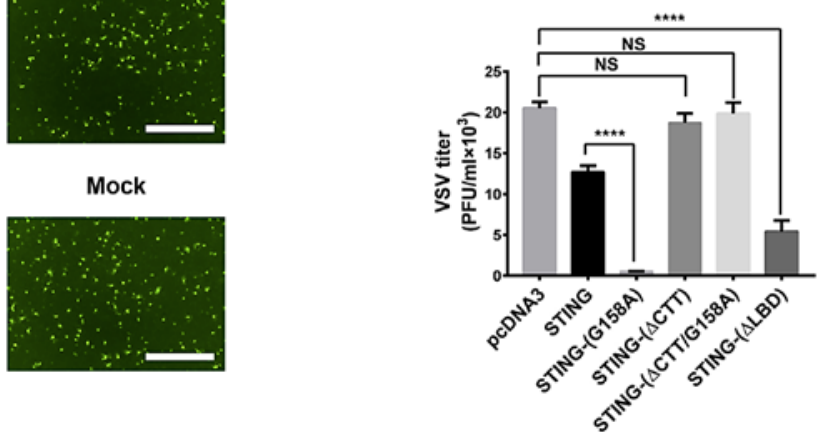

h

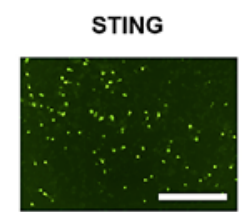

STING-(G158A)

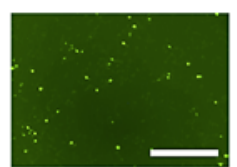

STING-( $\Delta C T T / G 158 A)$

STING-( $\triangle$ LBD)
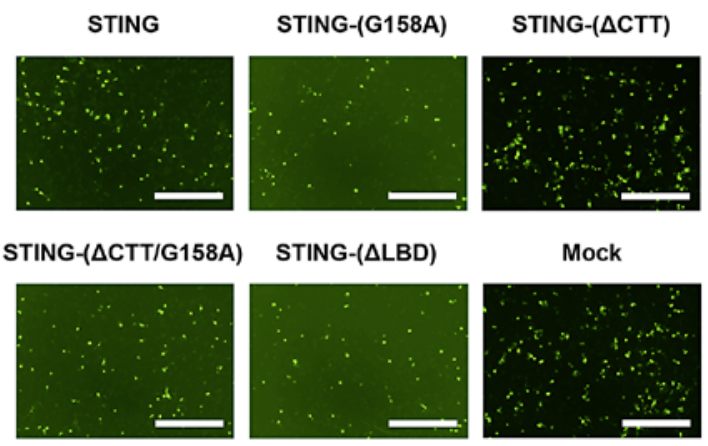

Mock
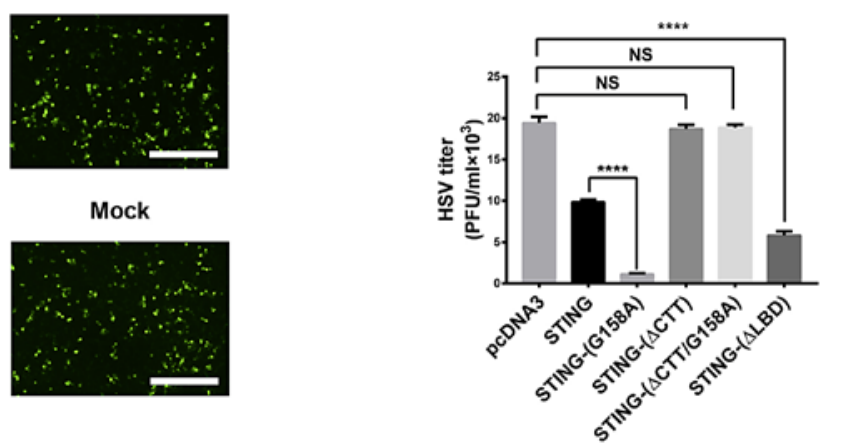

(For legend see next page.)

Human STING Is Regulated by an Autoinhibitory Mechanism
J Innate Immun 2022;14:518-531 DOI: $10.1159 / 000521734$ 


\section{Results}

\section{STING CTT Induces IFNB Expression}

STING contains three functional domains, including the N-terminal TM, cGAMP-binding LBD and CTT signaling domain (Fig. 1a). According to structural studies, binding to cGAMP triggers a $180^{\circ}$ rotation and following activation of STING LBD. A point mutation G158A could promote the rotation and cause STING activation, thereby bypassing the requirement of cGAMP binding. Utilizing this constitutively active mutant and deletion assays, we set out to determine specific signaling regions of STING for IFNB induction. Together with IFNB-luciferase reporter, STING was transiently expressed in HEK293T cells. As expected, wild type STING activated IFNB-luciferase reporter moderately, while STING(G158A) induced a more robust expression of IFNB-luciferase reporter (Fig. 1b). Meanwhile, quantitative PCR

Fig. 1. STING CTT can activate both IRF3 and NF- $\kappa B$. a Diagram depicting domain architectures of various forms of human STING. TM is shown in gray; LBD in blue, and CTT in yellow. STING$(\triangle \mathrm{CTT})$ and STING-( $\triangle \mathrm{LBD})$ are deletion mutants with $\mathrm{CTT}$ and LBD deleted, respectively. G158A is a point mutation substituting $\mathrm{G}$ at residue 158 with A. b Together with $I F N B$-luciferase reporter, various pcDNA3-flag-STING as indicated were transfected into HEK293T cells, respectively, for $24 \mathrm{~h}$. Luciferase activities were then measured. Protein expression levels are shown in online supplementary Figure 1a. c-e Various STING forms were transfected into HEK293T cells for $24 \mathrm{~h}$. The cells were then harvested and RNA was extracted. IFNB (c), ISG54 (d) and IL-6 (e) inductions were measured, respectively, by qPCR. $\mathbf{f}$ Low levels of STING and cGAS were transiently expressed in HEK293T cells for $24 \mathrm{~h}$. The cells were then infected with HSV-1-GFP for $12 \mathrm{~h}$, which were then harvested and total RNA was extracted. IFNB inductions were measured by qPCR. ISG54 and IL-6 inductions are shown in online supplementary Figure 1b. And protein expression levels are shown in online supplementary Figure 1c. g Various forms of STING were transfected into HEK293T cells for $24 \mathrm{~h}$. The cells were then infected with VSV-DM51-GFP. After $8 \mathrm{~h}$, fluorescent images were taken to visualize GFP-positive cells, indicating VSV-DM51-GFP proliferation (left). Scale bar, $10 \mu \mathrm{m}$. VSV-DM51-GFP titers were quantitated by plaque assay (right). h HEK293T cells expressing various STING forms were infected with HSV-1-GFP. After $12 \mathrm{~h}$, fluorescent images were taken to visualize GFP-positive cells, indicating HSV-1-GFP proliferation (left). Scale bar, $10 \mu \mathrm{m}$. HSV-1GFP titers were quantitated by plaque assay (right). STING in this study stands for human STING (hSTING) unless otherwise specified. Data information: All data are presented as the mean values based on three independent experiments, and error bars indicate SD. $p$ values were determined by unpaired two-tailed Student's $t$ test. ${ }^{*} p<0.05,{ }^{* *} p<0.01,{ }^{* * *} p<0.001$, and ${ }^{* * * *} p<0.0001$. NS indicates no statistically significant difference.
(qPCR) analysis also showed that STING-(G158A) stimulated IFNB expression markedly (Fig. 1c). Consistently, STING-(G158A) induced both ISG54 and interleukin IL-6 expression, suggesting two STING downstream signaling axes, IRF3 and NF- $\kappa \mathrm{B}$, were activated (Fig. 1d, e). Interestingly, a C-terminal truncation, STING-( $\triangle \mathrm{CTT})$, cannot stimulate either ISG54 or IL-6 expression, while an LBD deletion, STING- $(\triangle \mathrm{LBD})$, can induce ISG54 and $I L-6$ expression. To validate these results in a signal-dependent manner, we expressed STING together with cGAS at very low levels in HEK293T cells to avoid spontaneous activation. Upon HSV-1-GFP stimulation, IFNB were induced significantly in cells expressing either wild type STING or STING-(G158A) mutant but not in cells expressing STING mutants with CTT deletion (Fig. 1f). These data revealed that CTT harbors essential regions that are essential and sufficient for STING downstream IRF3 and NF- $\kappa$ B activation, indicative of full antiviral activity of STING-( $\triangle \mathrm{LBD})$. Indeed, HEK293T cells expressing STING-( $\triangle$ LBD) are resistant to both vesicular stomatitis virus (VSV-DM51GFP) and herpesvirus herpes simplex virus-1 (HSV-1-GFP) proliferation (Fig. 1g, h).

\section{STING CTT Fragment Artificially Localized to \\ Mitochondria Can Activate IRF3 and NF- $\kappa B$ \\ Signaling}

Structural studies revealed that binding to cGAMP is sufficient to trigger a conformational change of recombinant STING and its following activation. However, in cells, STING needs to traffic from ER to the Golgi to activate downstream signaling following cGAMP binding. One explanation is that only in Golgi can STING recruit downstream signaling molecules. To test the hypothesis, we fused STING CTT fragment to the TM of MAVS, a mitochondrial protein, so that STING CTT fragment will be targeted to mitochondria. A SUMO moiety is appended to this chimeric STING, namely STING(aa341-379)-mTM, to facilitate detection by Western blotting (Fig. 2a). As shown in Figure 2b, STING(aa-341379)-mTM can readily stimulate IFNB expression in HEK293T cells, while STING(aa-151-340)-mTM cannot induce IFNB expression, which lacks STING signaling CTT domain. In consistence, STING(aa-341-379)mTM can induce ISG54 and $I L-6$ expression, indicating the full activation of STING downstream signaling pathways (Fig. 2c, d). Remarkably, expression of STING(aa341-379)-mTM renders HEK293T cells resistant to VSV-DM51-GFP and HSV-1-GFP infection, suggesting the STING CTT can execute full antiviral function from 
a

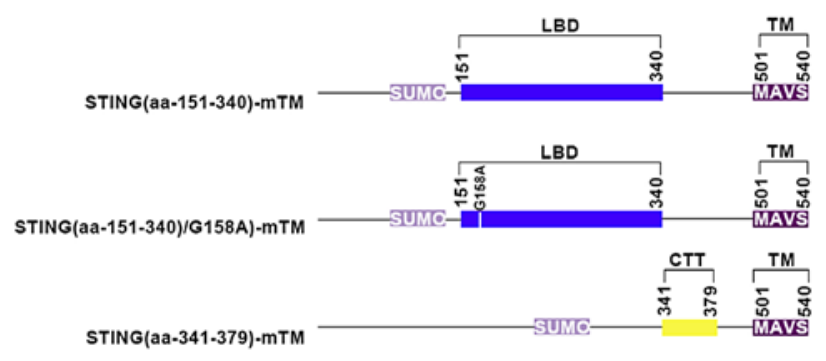

b

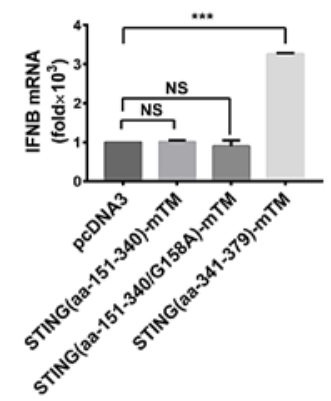

C

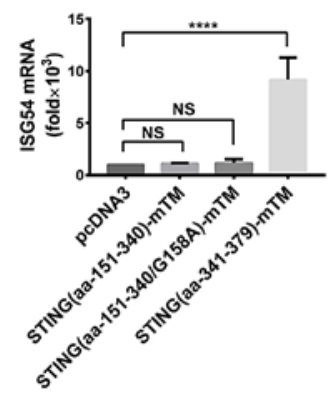

e
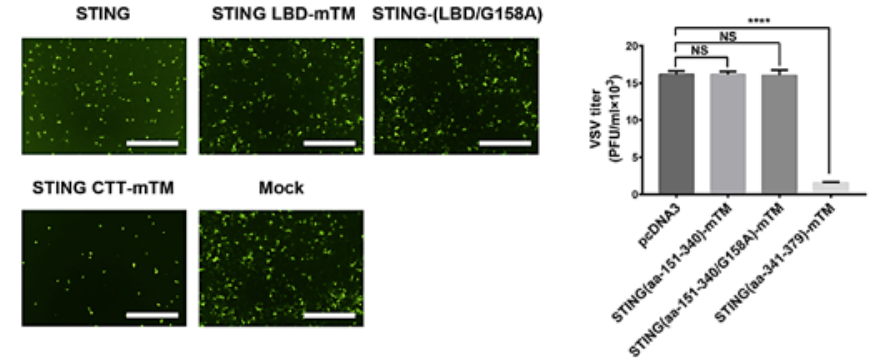

d

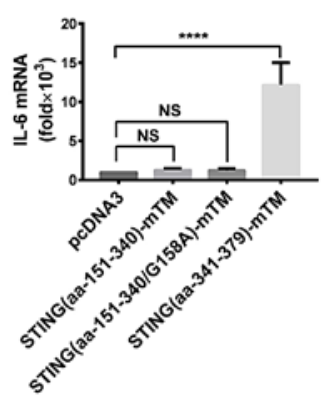

f
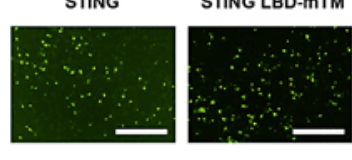

Mock
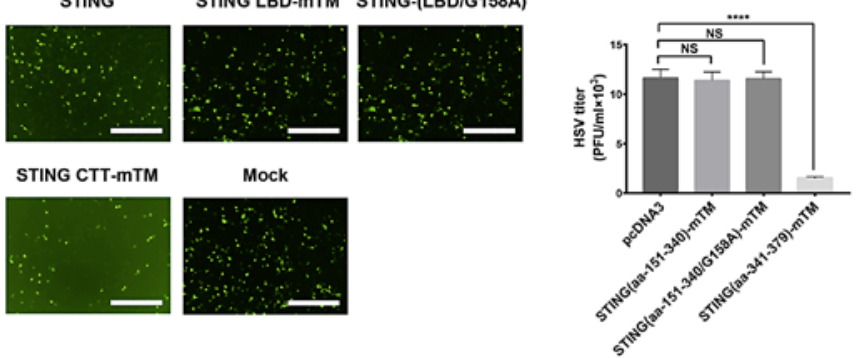

g
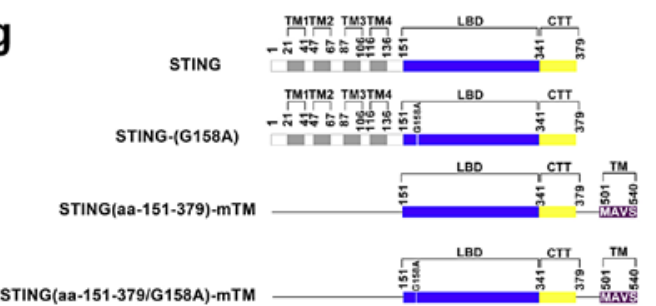

h
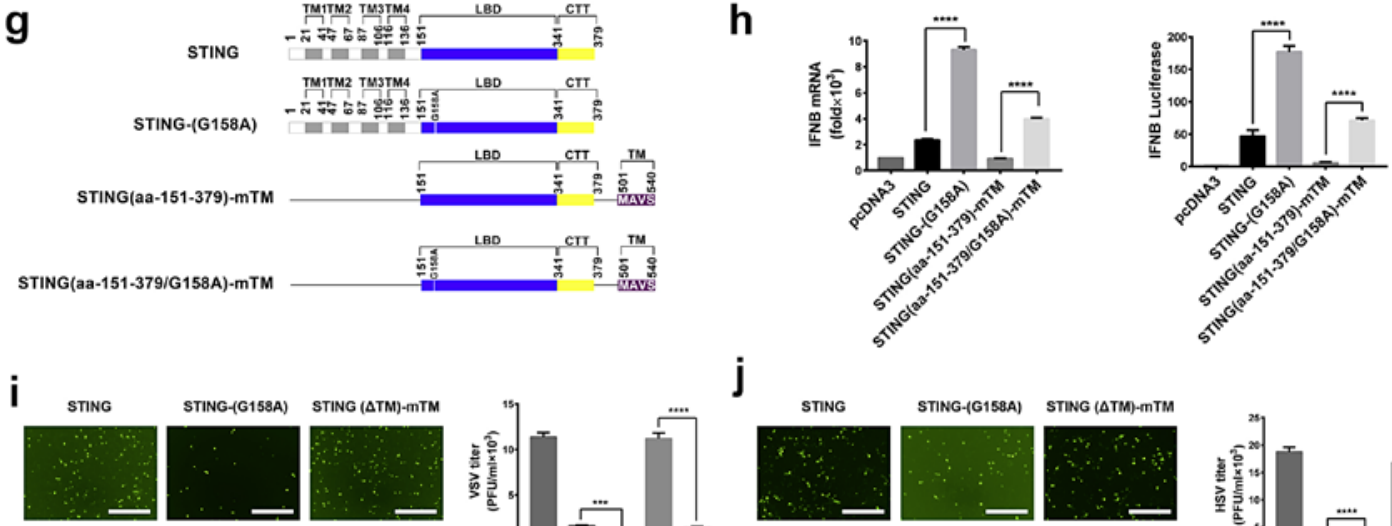

STINO(ATWO15AA)-mTM

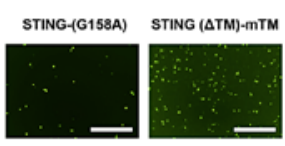

Mock
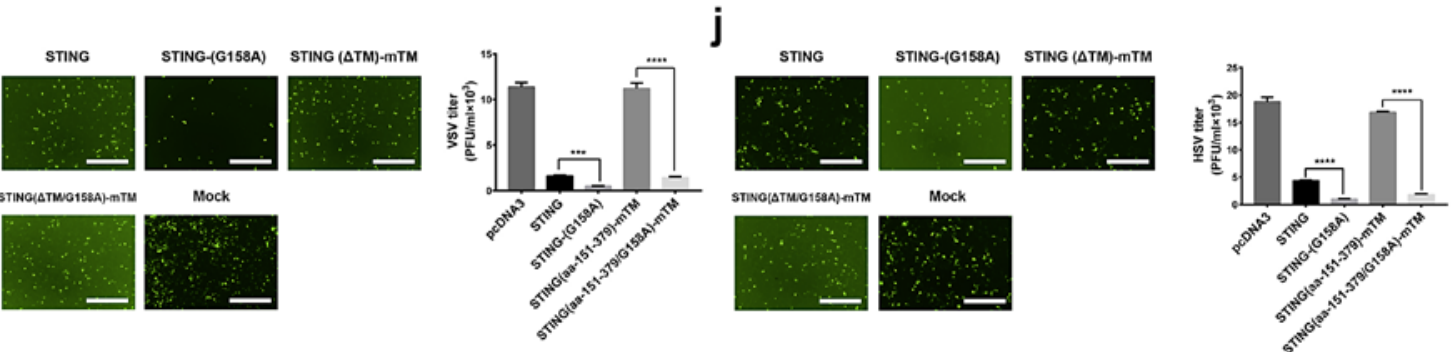

(For legend see next page.)

Human STING Is Regulated by an Autoinhibitory Mechanism
J Innate Immun 2022;14:518-531 DOI: $10.1159 / 000521734$ 
mitochondria (Fig. 2e, f). In addition, we made another mitochondria-anchored STING mutant containing its LBD and CTT, i.e., STING(aa-151-379)-mTM (Fig. 2g), A G158A point mutation in this chimeric STING stimulates IFNB expression pronouncedly, which is reminiscent of ER-localized STING (Fig. 2h). As a result, STING(aa-151-379/G158A)-mTM renders HEK293T cells much more resistant to virus infection than STING(aa-151-379)-mTM (Fig. 2i, j). Taken together, these data indicated that STING CTT can signal to

Fig. 2. STING CTT fragment can activate both IRF3 and NF- $\kappa B$ from mitochondria. a Diagram illustrating protein expression constructs. STING(aa-151-340)-mTM is a chimeric form of STING LBD (aa-151-340) fused with MAVS TM (aa-501-540). G158A is a point mutation replacing $G$ with A. STING(aa-341379)-mTM is a fusion form of STING CTT (aa-341-379) and MAVS TM (aa-501-540). STING LBD is shown in blue, STING CTT in yellow, and MAVS TM in purple. b-d Various STING forms were transfected into HEK293T cells for $24 \mathrm{~h}$. The cells were then harvested and IFNB (b), ISG54 (c), and IL-6 (d) inductions were measured, respectively, by qPCR. Protein expression levels are shown in online supplementary Figure 2a. e Various forms of STING were transfected into HEK293T cells for $24 \mathrm{~h}$, followed by infection with VSV-DM51-GFP. Fluorescent images were taken 8 $\mathrm{h}$ after VSV-DM51-GFP infection to visualize GFP-positive cells (left), and VSV-DM51-GFP proliferation was quantified (right). Scale bar, $10 \mu \mathrm{m}$. f HEK293T cells were transfected with constructs as indicated, followed by HSV-1-GFP infection. Fluorescent images were taken $12 \mathrm{~h}$ after HSV-1-GFP infection to examine HSV1-GFP proliferation (left). Scale bar, $10 \mu \mathrm{m}$. HSV-1-GFP titers were quantitated by plaque assay (right). g A diagram illustrating various forms of STING. STING(aa-151-379)-mTM is the chimeric form of STING(aa-151-379) and MAVS TM (aa-501-540). G158A is a point mutation replacing $G$ with A. $\mathbf{h}$ Expression vectors as indicated were transfected into HEK293T cells, and both endogenous IFNB and IFNB-luciferase inductions were measured $24 \mathrm{~h}$ after transfection. Protein expression levels are shown in online supplementary Figure 2b. i Various expression constructs were transfected into HEK293T cells. Twenty-four hours after transfection, HEK293T cells were infected with VSV-DM51-GFP. Fluorescent images were taken $8 \mathrm{~h}$ after VSV-DM51-GFP infection to visualize GFP-positive cells, indicating VSV-DM51-GFP proliferation (left). Scale bar, $10 \mu \mathrm{m}$. VSV-DM51-GFP titers were quantitated by plaque assay (right). $\mathbf{j}$ After transfection with various expression constructs, HEK293T cells were infected with HSV-1-GFP for $12 \mathrm{~h}$. Fluorescent images were taken to visualize GFP-positive cells, indicating HSV-1-GFP proliferation (left). Scale bar, $10 \mu \mathrm{m}$. HSV-1-GFP titers were quantitated by plaque assay (right). Data information: All data are presented as the mean values based on three independent experiments, and error bars indicate SD. $p$ values were determined by unpaired two-tailed Student's $t$ test. ${ }^{*} p<0.05,{ }^{* *} p<0.01,{ }^{* * *} p<0.001$, and ${ }^{* * * *} p<0.0001$. NS indicates no statistically significant difference. downstream molecules to induce IFNB expression from mitochondria. In the other word, Golgi compartment is not the only site for STING to recruit downstream signaling molecules. Therefore, we deduce that the traffic of STING from ER to Golgi upon cGAMP-binding in cells might implicate important events other than recruiting downstream signaling molecules.

\section{Identification of a STING Region (aa-345-357) as a \\ Specific NF- $\kappa B$-Stimulating Region}

We then investigated how STING CTT might activate both IRF3 and NF- $\mathrm{B}$ signaling. STING CTT contains less than 40 residues, in which a PLPLRT/SD motif was found to mediate TBK1 recruitment and following IRF3 phosphorylation. We hypothesized that TBK1, which shares high homology to IKK, might be also involved in NF- $\kappa \mathrm{B}$ activation. Therefore, we tried to determine if STING can activate NF- $\kappa B$ in TBK1-deficient cells. In TBK1 KO HEK293T cells, overexpressed STING cannot induce ISG54 expression, suggesting that IRF3 axis cannot be activated as expected. On the contrary, IL- 6 can be induced readily by STING in TBK1 KO cells, indicating TBK1 might not be involved in NF- $\kappa \mathrm{B}$ activation and the region for NF- $\mathrm{\kappa B}$ activation might be different from PLPLRT/SD motif or TBK1-binding motif (online suppl. Fig. 3a). These results were also validated in a stimulation-dependent manner when cGAS and STING were expressed at very low levels to prevent spontaneous activation (Fig. 3a). We then went on to determine the region of STING CTT specifically for NF$\kappa \mathrm{B}$ activation. A pLxIS motif in STING CTT was reported to mediate IRF3 docking and following activation (Fig. 3b). A mutation in pLxIS motif disrupted the activity of STING(CTT/I365A/S366A)-mTM to stimulate ISG54 expression (Fig. 3c). Unexpectedly, STING(CTT/ I365A/S366A)-mTM can still induce $I L-6$ expression, suggesting STING CTT harbors a region for NF- $\kappa \mathrm{B}$ activation that is independent of the pLxIS motif. We then made some deletions to map the region specific for IRF3 and NF- $\mathrm{KB}$ activation, respectively. STING(aa-358379)-mTM can stimulate ISG54 expression but not $I L-6$ expression, indicating STING region aa-358-379 is specific for IRF3 but not NF- $\kappa \mathrm{B}$ activation (Fig. $3 \mathrm{c}$ ). In contrast, STING(aa-341-357)-mTM induces IL-6 expression as robust as STING(aa-341-379)-mTM, indicating STING aa-341-357 covers the region for NF- $\kappa B$ activation (online suppl. Fig. 3d, e). STING(aa-341-357)mTM cannot induces ISG54 expression, suggesting this region is only for NF- $\kappa B$ but not IRF3 activation. Moreover, we determined that region aa-345-357 is sufficient 
a
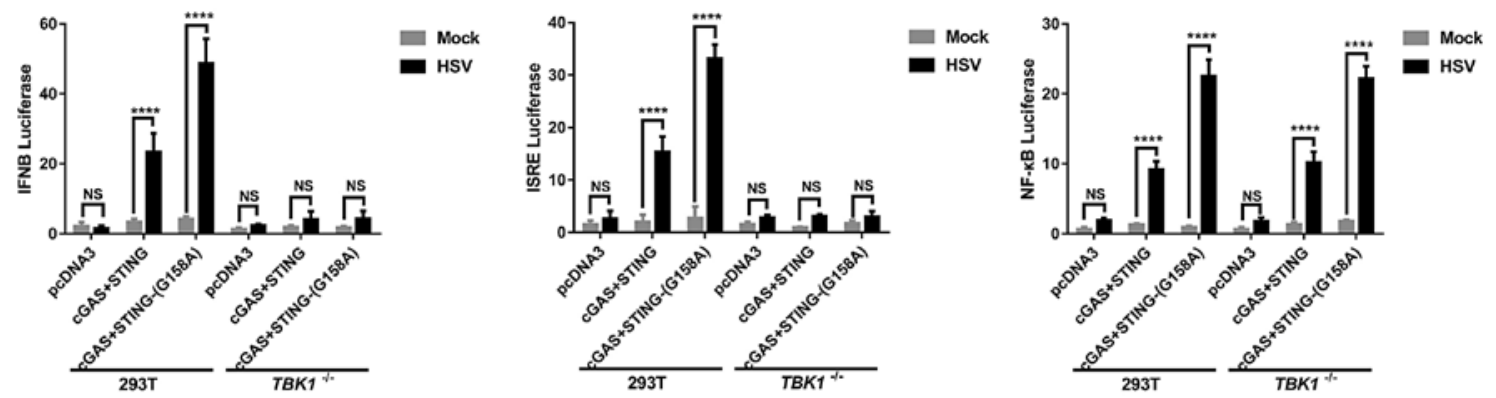

b

ڤ IRF3 ๕

Human VTVGS LKTSA VPSTS TM SQE PELLI ŜMMEK PLPLR TDFS

Mouse VTMNA PMTSV APPPS VL SQE PRLLI SGMDQ PLPLR TDLI

STING(CTT/I365A/S366A)-mTM —IN

C

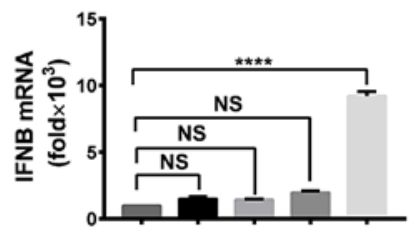

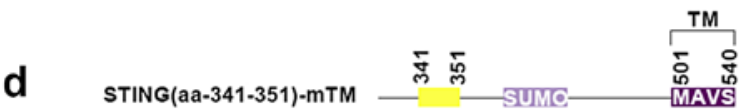

STING(aa-345-357)-mTM —

STING(aa-350-357)-mTM L

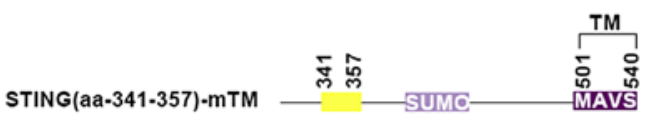

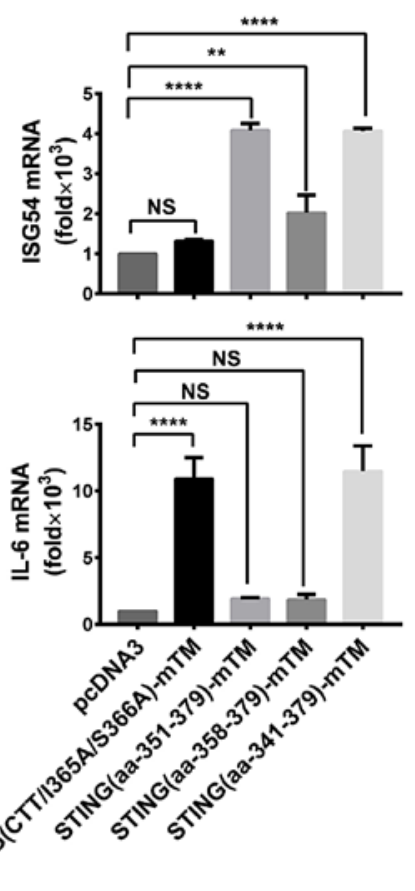

e
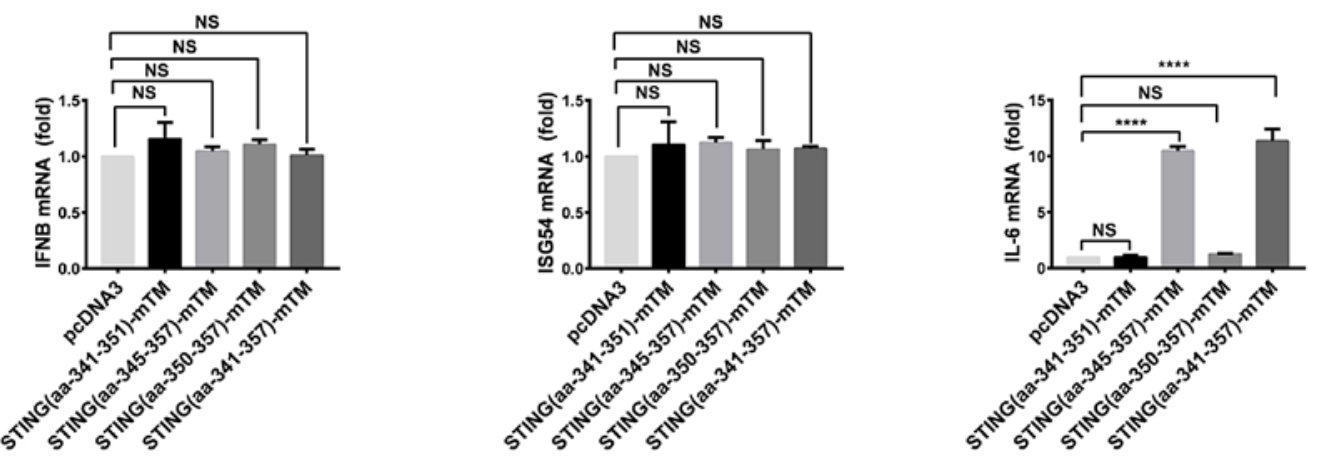

(For legend see next page.)

Human STING Is Regulated by an Autoinhibitory Mechanism
J Innate Immun 2022;14:518-531 DOI: $10.1159 / 000521734$ 
for NF- $\kappa \mathrm{B}$ activation (Fig. 3d, e). When fused with a peptide from VAMP-2 for targeting ER localization, this region (STING-aa-345-357) can activate NF-kB- but not IRF3-signaling (Fig. 4a). Furthermore, in the context of cGAS-cGAMP-dependent activation, we found that STING region aa-345-357 is indeed required for NF- $\mathrm{kB}$ but not IRF3 activation (Fig. 4b). By examining the sequence alignment of STING from seven different species, we found that the NF- $\mathrm{kB}$-activating region of hSTING is highly conserved in mammals, including human, monkey, dog, pig and mouse, while it is not conserved in chicken or zebra fish (Fig. 4c). A recent report showed that zebra fish STING-CTT can recruit TRAF6 and activate NF- $\kappa$ B. As expected, zebra fish STING (zSTING) can activate NF-kB in HEK293T cells and zSTING with CTT deletion ( $\triangle$ aa-381-398) can no longer activate NF$\kappa \mathrm{B}$ (Fig. 4d). Additionally, we made a chimeric STING, namely, zSTING( $\Delta$ CTT)-hSTING(aa-345-357), in which zSTING CTT was replaced by the NF- $\kappa B-$ activating region of hSTING. This chimeric STING could not activate either IRF3 or NF- $\kappa \mathrm{B}$ signaling, suggesting that the region leading zSTING CTT might confer inhibition on the hSTING CTT (Fig. 4d).

Fig. 3. STING region aa-341-357 can activate NF- $\kappa B$ specifically. a STING- and cGAS-expressing vectors together with IFNB-luciferase reporter construct (left), ISRE-luciferase reporter construct (middle), or $N F-\kappa B$-luciferase reporter construct (right) were transfected into wild-type HEK293T or TBK1 knockout HEK293T for $24 \mathrm{~h}$, respectively. Firefly luciferase inductions were measured after infection with HSV-1-GFP for $12 \mathrm{~h}$. Protein expression levels are shown in online supplementary Figure 3b. b A diagram illustrating various deletions or mutants based STING(aa-341-379)mTM as described in Figure 2a. STING(CTT/I365A/S366A)mTM harbors double mutations (with I to A at position 365 and $S$ to A at position 366). c Various STING(aa 341-379)-mTM deletions and mutants were transfected into HEK293T cells. Twentyfour hours post transfection, the cells were collected and IFNB, ISG54, and IL-6 were measured, respectively, by qPCR. Protein expression levels are shown in online supplementary Figure $3 \mathrm{c}$. d A diagram illustrating various STING (aa-341-379)-mTM deletions. e STING(aa 341-379)-mTM deletions as indicated were transfected into HEK293T cells. Twenty-four hours post transfection, the cells were collected and IFNB, ISG54, and IL-6 inductions were measured, respectively, by qPCR. Protein levels were analyzed by Western blot in online supplementary Figure 3g. Data information: All data are presented as the mean values based on three independent experiments, and error bars indicate SD. $p$ values were determined by unpaired two-tailed Student's $t$ test. ${ }^{*} p<$ $0.05,{ }^{* *} p<0.01,{ }^{* * *} p<0.001$, and ${ }^{* * * *} p<0.0001$. NS indicates no statistically significant difference.
An Autoinhibitory Mechanism Regulates STING CTT Activity

As demonstrated in Figure $1 \mathrm{~b}$, a point mutation STING(G158A) resulted in dramatically elevated induction of IFNB, circumventing cGAMP-binding in STING activation. Strikingly, the same single mutation also led to activation of mitochondria-localized STING, i.e., STING(aa-151-379/G158A)-mTM, which does not respond to cGAS-cGAMP signaling (Fig. 5a). Mechanistically, the G158A mutation triggered a $180^{\circ}$ rotation of LBD in contrast to wild type STING, leading to an overall active conformation of STING. However, how CTT, the signaling domain gets activated as a result of the conformational change of LBD is unknown. We speculate that there are two possible mechanisms. One possibility is that CTT switches from an inactive to active conformation as LBD rotates. The abovementioned data showed that STING- $(\triangle \mathrm{LBD})$ can activate both IRF3 and NF- $\kappa$ B regardless of the presence of LBD (Fig. 1d, e), which argues against that the active conformation of STING CTT depends on its LBD. The other possibility is that CTT can form an active conformation by itself and is released from an autoinhibition by LBD during the activation process. In supporting of the latter scenario, STING CTT fragment showed strong association with its LBD in our coimmunoprecipitation assay (Fig. 5b). Taken together, we conclude that LBD might modulate CTT activity in an autoinhibitory manner through direct interaction.

Our abovementioned data suggested that STING LBD might bind to and inhibit its CTT in an intramolecular manner, we then went on to test whether STING LBD can inhibit its CTT signaling in trans. Both LBD and CTT fragments were expressed transiently in HEK293T cells. As expected, STING- $(\Delta \mathrm{LBD})$ alone stimulates IFNB expression readily. In contrast, coexpression of STING- $(\triangle \mathrm{CTT})$ can abolish the IFN-inducing activity of STING-( $\triangle \mathrm{LBD})$, suggesting that STING LBD can indeed inhibit CTT signaling in trans (Fig. 5c). Additionally, mitochondrion-localized STING(aa-151340)-mTM can disrupt IFNB induction by STING(aa341-379)-mTM, confirming the inhibitory effect of STING LBD on CTT activity (Fig. 5c; online suppl. Fig. $4 d)$. These data also suggested that STING LBD can inhibit CTT activity independently of the ER localization. Furthermore, we tested whether STING LBD can inhibit endogenous STING in HEK293 cells, which express both endogenous cGAS and STING in contrast to HEK293T cells (online suppl. Fig. 4c). We transiently expressed STING LBD fragment in HEK293 cells with or without virus infection. Indeed, STING- $(\Delta \mathrm{CTT})$ dis- 
a

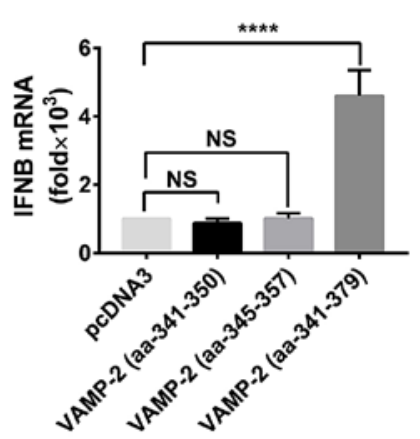

b

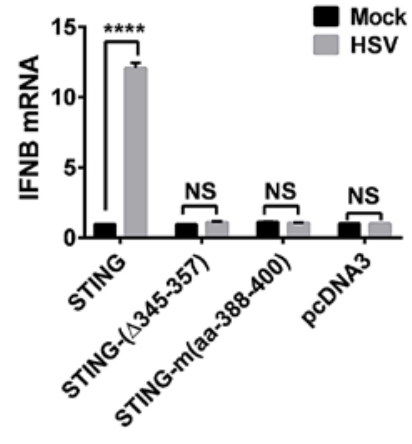

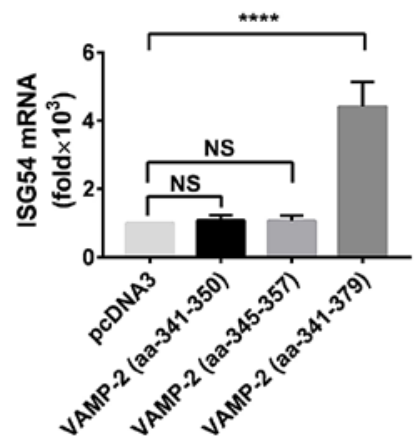

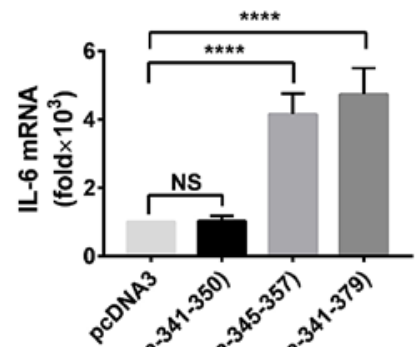

$3^{x} \quad 3^{x x^{5}}$

Human VTVGS LKTSA VPSTS

Monkey VTVGS LKNSA VPSTS

pig VTMGS AETSV VPTSS

Dog VTMGS MDTSI VPTSS

Mouse VTMNA PMTSV APPPS
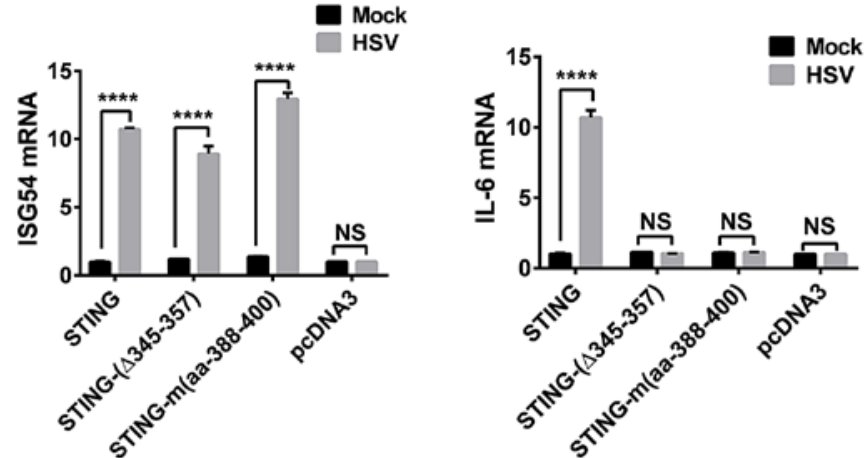

${ }_{3}^{51} \quad$ pLxIS
TBM $\quad 3^{19}$

TMSQE PELLI SGMEK PLPLR TDFS TMSOE PELLI SGMEK PLPLR TDFS TLSQE PELLI SGMEQ PLPLR SDIF TLSQE PNLFI SGLEQ PLPLR TDIF VISQE PRLLI SGMDQ PLPLR TDLI

TGSVE LSLQV SSSDL PQPLR SDCP

Chicken YMVQE EL--- --PLG PMSDE PTLMF S---R PQSLR SEPV

d
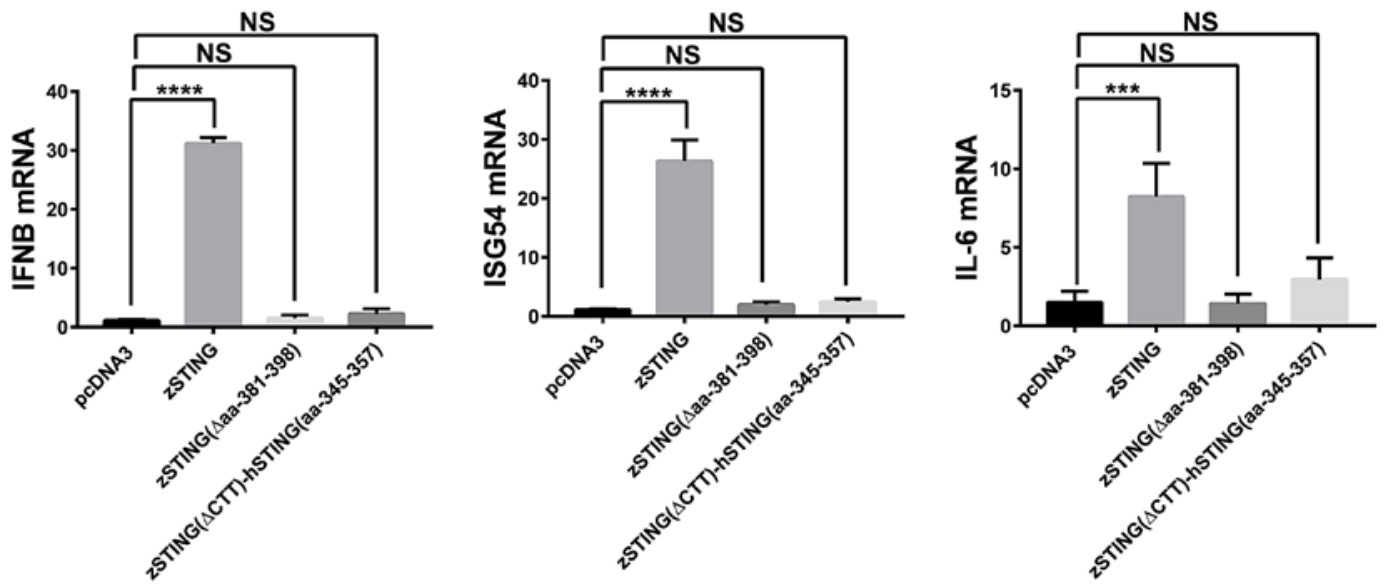

(For legend see next page.)

Human STING Is Regulated by an Autoinhibitory Mechanism
J Innate Immun 2022;14:518-531 DOI: $10.1159 / 000521734$ 
rupted IFNB induction in HEK293 cells in response to HSV-1-GFP stimulation, suggesting that STING LBD fragment might interfere with endogenous STING activation in cGAS pathway (Fig. 5d). Expression of STING$(\triangle \mathrm{CTT})$ showed no detectable effect on IFNB induction in HEK293 cells in response to VSV-DM51-GFP infection, indicating that STING LBD fragment might not interact with RIG-I-MAVS pathway. Altogether, these data suggested that STING LBD fragment could play a dominant negative role in regulating endogenous STING upon upstream stimulation.

\section{Discussion}

In this study, we identify that a region in STING CTT (aa-345-357) serves as a platform specifically for NF- $\kappa B$ activation, which might be the last piece of information for the linear arrangement of functional domains of human STING. We find that STING CTT can activate both IRF3 and NF- $\kappa B$ to induce IFN-I expression even if enforced to be localized to mitochondria, suggesting that STING signaling domain could recruit downstream sig-

Fig. 4. Human STING region aa-345-357 can activate NF- $\kappa B$ when fused with a targeting peptide but not when fused with a zebra fish STING fragment. a Various STING CTT deletions fused with a peptide from VAMP-2 were transiently expressed in HEK293T cells for $24 \mathrm{~h}$. The cells were then harvested and IFNB (left), ISG54 (middle), and IL-6 (right) inductions were measured, respectively, by qPCR. Protein expression levels are shown in online supplementary Figure 4a. b Various STING forms were transiently expressed in HEK293T cells at low level for $24 \mathrm{~h}$, followed by infection with HSV-1-GFP for $12 \mathrm{~h}$. The cells were then harvested and IFNB (left), ISG54 (middle), and IL-6 (right) inductions were measured, respectively, by qPCR. STING-m(aa-388-400) was generated with a replacement of hSTING region aa-345-357 with MAVS region aa-388-400. c Sequence alignment of STING CTT from different species. The NF- $\mathrm{kB}$-activating region aa-388400 of hSTING, as well as pLxIS motif and TBMs, was highlighted. The numbers indicated residues of hSTING. d Various STING forms were transfected into HEK293T cells for $24 \mathrm{~h}$. The cells were then harvested and IFNB (left), ISG54 (middle), and IL-6 (right) inductions were measured, respectively, by qPCR. Protein expression levels are shown in online supplementary Figure $4 \mathrm{~b}$. zSTING $(\triangle$ CTT aa-381-398) is a deletion of zebra fish STING. zSTING( $\Delta$ CTT)-hSTING(aa-345-357) is a chimeric form of zebra fish STING( $\Delta$ aa-381-398) fused with human STING(aa-345-357). Data information: All data are presented as the mean values based on three independent experiments, and error bars indicate SD. $p$ values were determined by unpaired two-tailed Student's $t$ test. ${ }^{*} p<0.05,{ }^{* *} p<0.01,{ }^{* * *} p<0.001$, and ${ }^{* * * *} p<0.0001$. NS indicates no statistically significant difference. TBM, TBK1-binding motif. naling molecules from organelles other than ER or Golgi. Lastly, we uncover STING LBD can bind to CTT and provides autoinhibition for the signaling domain, which can be unlocked by cGAMP binding.

Transcriptional factors IRF3 and NF- $\kappa \mathrm{B}$ are two essential axes of IFN-I induction in innate immune response. In contrast to the elegant mechanisms underlying TBK1 and IRF3 activation, little is known on NF- $\kappa B$ activation by STING. A recent report suggests that TBK1 mediates NF- $\kappa B$ activation by STING based on the data that a L373A mutation disrupts $I L-6$ induction [33]. In contrast, our data showed that the TBK1-binding region (aa-371-376) of STING is dispensable for $\mathrm{NF}-\kappa \mathrm{B}$ activation, suggesting TBK1 is not required for $\mathrm{NF}-\kappa \mathrm{B}$ activation. In fact, we identified a novel region aa-345-357 for specific NF- $\kappa B$ activation by STING. This NF- $\kappa B$-activating region is independent of IRF3 activation. Both regions are within STING CTT, constituting the linear layout of STING signaling domains. In human STING, the NF- $\kappa \mathrm{B}$-activating region sits right in front of IRF3-activating region, in stark contrast to the recently identified NF- $\kappa \mathrm{B}$-activating region from zebra fish, which is a CTT extension [28]. Interestingly, the NF- $\mathrm{kB}$-activating region of hSTING is not conserved in zebra fish STING. Substitution of zebra fish STING CTT with the NF- $\kappa \mathrm{B}$-activating region of hSTING does not confer signaling activity on zSTING, suggesting a divergent regulatory mechanism underlying STING activation evolutionarily. The remaining question is how this NF- $\kappa \mathrm{B}$-activating region of hSTING might engage IKK and possibly TRAF family members for NF- $\kappa \mathrm{B}$ activation as MAVS does, which is worth further investigation.

One of the puzzling mechanisms underlying STING activation is its traffic from ER to Golgi upon cGAMPbinding. One explanation is that STING might only be able to recruit downstream signaling molecules from Golgi. To test this possibility, we engineered a chimeric STING, in which human STING signaling domain is fused with MAVS TM so that it is localized to mitochondria rather than ER or Golgi. Strikingly, the chimeric STING can stimulate downstream signaling and induce IFNB production robustly, indicating that STING does not have to be on ER or Golgi to recruit downstream signaling molecules. Another possibility is that STING can gain full polymerization and activation following traffic to Golgi, and in this case, factors promoting full activation of STING warrant further study. As a matter of fact, a recent report showed that Golgi apparatus-synthesized sulfated glycosaminoglycans mediated STING 
a

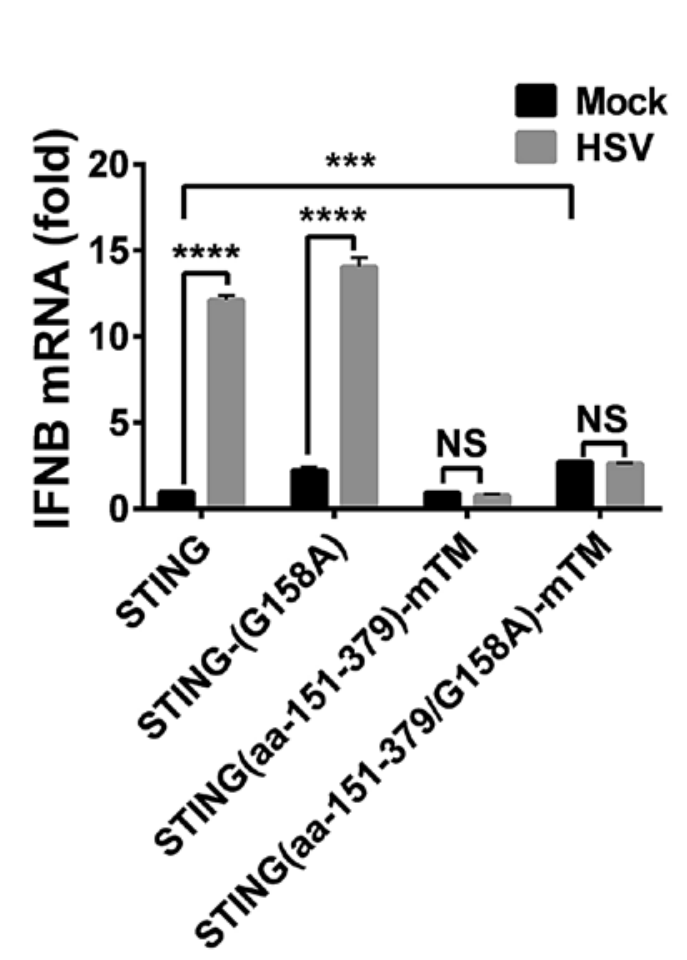

b $\begin{array}{lllll}\text { FLAG-STING-(ALBD) } & + & - & - & - \\ \text { FLAG-STING-(ACTT) } & - & + & - & - \\ \text { HA.STING-(ALBD) } & - & + & - & + \\ \text { HA-STING-(ACTT) } & + & - & + & - \\ \text { FLAG.TM-SUMO } & - & - & + & +\end{array}$

Kda

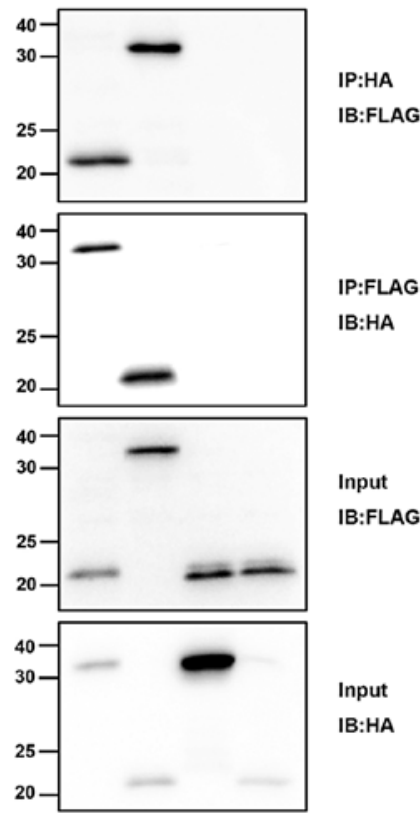

C

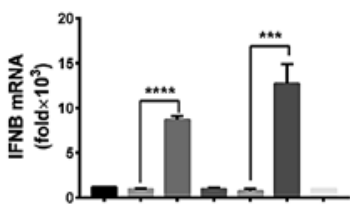

STING- $\triangle \mathrm{CTT})++-\quad-\quad-$

STING- $\triangle$ LBD) - + + - - -

STING(aa-151-340)-mTM - - - + + - -

STING(aa-341-379)-mTM - - - - + + -

pcDNA3 + - + + + +

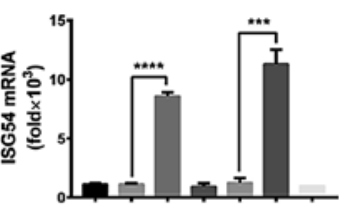

$\operatorname{STING}-(\triangle \mathrm{CTT})++\quad-\quad-\quad \cdot$

$\operatorname{STING}-(\triangle \mathrm{LBD})-++-\cdot-\cdot$

STING(aa-151-340)-mTM - - - + + - -

STING(aa-341-379)-mTM $-\quad \cdot-\quad+\quad+$

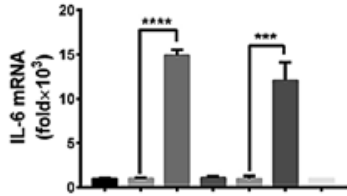

$\operatorname{STING}-(\triangle \mathrm{CTT})++-\quad \cdot-\cdot$

STING- $\triangle$ LBD) - + + - - -

STING(aa-151-340)-mTM - - - + + - -

STING(aa-341-379)-mTM - - - - + + -

pcDNA3 + - + + + + d

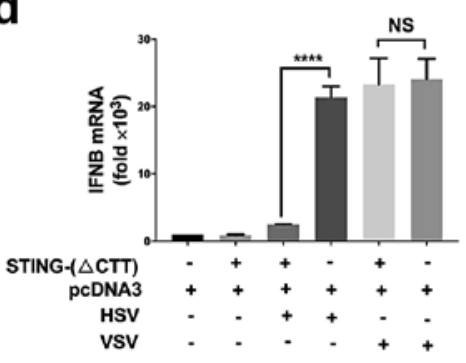

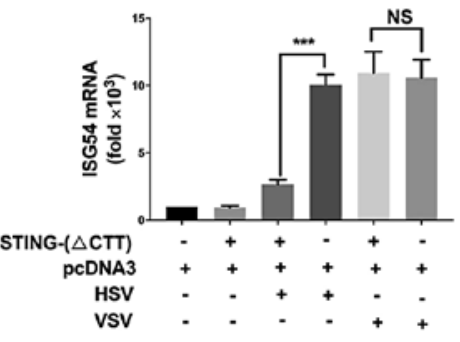

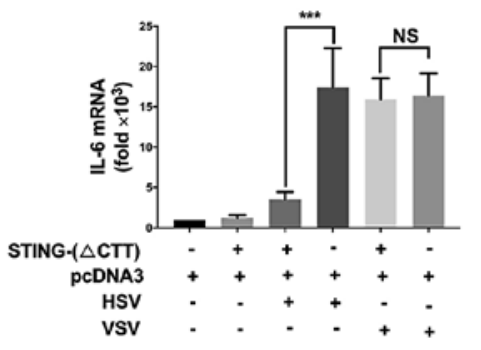

5

(For legend see next page.)

Human STING Is Regulated by an Autoinhibitory Mechanism
J Innate Immun 2022;14:518-531 DOI: $10.1159 / 000521734$ 
activation. In this regard, more thorough mechanistic study might be expected.

STING-associated vasculopathy with onset in infancy is associated with STING mutations, which lead to its spontaneous activation and accidental induction of IFN-I [34-37]. Mechanistically, these mutations may trigger a conformational change of STING LBD, mimicking the consequence resulting from cGAMP binding. However, the mechanism underlying the activation of STING signaling domain CTT following the conformational change of LBD is unknown. A previous report showed that CTT inhibits LBD aggregation and STING activity [38]. In contrast, our data showed that STING CTT alone can induce downstream IRF3 and NF- $\mathrm{kB}$ activation. We further found that LBD binds to CTT and provide a sophisticated autoinhibitory mechanism regulating STING activity in the absence of upstream signal. Upon infection, cGAMP binds to STING, which triggers the conformational change of LBD and releases the CTT for downstream signaling. The autoinhibitory mechanism regulating STING activity resembles the intramolecular autoinhibition modulating MAVS activity in RIG-I pathway.

Fig. 5. STING LBD fragment can inhibit CTT signaling activity in an intramolecular and intermolecular manner. a Low amount of plasmids expressing STING and cGAS were transfected into HEK293T cells. The cells were infected with HSV-1-GFP $24 \mathrm{~h}$ after transfection. Twelve hours post infection, the cells were harvested and IFNB induction was measured by qPCR. b Plasmids expressing FLAG- or HA-tagged STING- $(\triangle \mathrm{CTT})$ and STING-( $\triangle \mathrm{LBD})$ were transfected into HEK293T cells. After $48 \mathrm{~h}$, cells were harvested and immunoprecipitations were performed, followed by immunoblotting. c Various expression constructs were transfected into HEK293T cells and IFNB (left), ISG54 (middle) and IL-6 induction (right) were measured, respectively, by qPCR. STING$(\triangle \mathrm{CTT})$ and STING-( $\triangle \mathrm{LBD})$ are described in Figure 1a. STING(aa151-340)-mTM and STING(aa-341-379)-mTM are described in Figure 2a. Protein expression levels are shown in online supplementary Figure 4d. d Various constructs as indicated were transfected into HEK293 cells. Twenty-four hours after transfection, the cells were infected with VSV-DM51-GFP or HSV-1-GFP. Eight hours post infection with VSV-DM51-GFP and $12 \mathrm{~h}$ post infection with HSV-1-GFP, RNA was extracted and IFNB, ISG54, and IL-6 induction were measured by qPCR, respectively. Protein expression levels are shown in online supplementary Figure 4e. Data information: All data are presented as the mean values based on three independent experiments, and error bars indicate SD. $p$ values were determined by unpaired two-tailed Student's $t$ test. ${ }^{*} p<0.05$, ${ }^{* *} p<0.01,{ }^{* * *} p<0.001$, and ${ }^{* * * *} p<0.0001$. NS indicates no statistically significant difference.

\section{Acknowledgments}

We thank all members of Hou Lab in Shanghai Institute of Biochemistry and Cell Biology for technical support and discussions.

\section{Statement of Ethics}

This study only involves some cell lines used by most of the labs in the field, which does not require an approved protocol.

\section{Conflict of Interest Statement}

The authors have no competing interest to declare.

\section{Funding Sources}

This work is supported by funding from the National Natural Science Foundation of China (32070902) and the Chinese Academy of Sciences Pilot Strategic Science and Technology Project B (XDB37030207) to F.H.

\section{Author Contributions}

S.Q. led the study and performed most of the experiments. C.W. provided imaging data and results on zebra fish STING. R.Z. employed the study in TBK1-deficient cells. J.Z., X.H., Y.J., J.L., and M.R. contributed with technical help and discussions. F.H. and M.L. designed and organized the study.

\section{Data Availability Statement}

All data generated or analyzed during this study are included in this article and its online supplementary material. Further inquiries can be directed to the corresponding author.

\section{References}

1 Aoshi T, Koyama S, Kobiyama K, Akira S, Ishii KJ. Innate and adaptive immune responses to viral infection and vaccination. Curr Opin Virol. 2011;1(4):226-32.

2 Takeuchi O, Akira S. Pattern recognition receptors and inflammation. Cell. 2010;140(6): 805-20.

3 Zhang X, Bai XC, Chen ZJ. Structures and mechanisms in the cGAS-STING innate immunity pathway. Immunity. 2020;53(1):4353.

4 Ablasser A, Hur S. Regulation of cGAS- and RLR-mediated immunity to nucleic acids. Nat Immunol. 2020;21(1):17-29.

5 Chow KT, Gale M Jr, Loo YM. RIG-I and other RNA sensors in antiviral immunity. Annu Rev Immunol. 2018;36:667-94. 
6 Chen Q, Sun L, Chen ZJ. Regulation and function of the cGAS-STING pathway of cytosolic DNA sensing. Nat Immunol. 2016;17(10): 1142-9.

7 Wu J, Sun L, Chen X, Du F, Shi H, Chen C, et al. Cyclic GMP-AMP is an endogenous second messenger in innate immune signaling by cytosolic DNA. Science. 2013;339(6121):82630.

8 Sun L, Wu J, Du F, Chen X, Chen ZJ. Cyclic GMP-AMP synthase is a cytosolic DNA sensor that activates the type i interferon pathway. Science. 2013;339(6121):786-91.

9 Sun W, Li Y, Chen L, Chen H, You F, Zhou X, et al. ERIS, an endoplasmic reticulum IFN stimulator, activates innate immune signaling through dimerization. Proc Natl Acad Sci U S A. 2009;106(21):8653-8.

10 Ishikawa $\mathrm{H}, \mathrm{Ma} \mathrm{Z}$, Barber GN. STING regulates intracellular DNA-mediated, type I interferon-dependent innate immunity. $\mathrm{Na}$ ture. 2009;461(7265):788-92.

11 Zhong B, Yang Y, Li S, Wang YY, Li Y, Diao $\mathrm{F}$, et al. The adaptor protein MITA links virus-sensing receptors to IRF3 transcription factor activation. Immunity. 2008;29(4):53850.

12 Shang G, Zhang C, Chen ZJ, Bai X-C, Zhang $X$. Cryo-EM structures of STING reveal its mechanism of activation by cyclic GMPAMP. Nature. 2019;567(7748):389-93.

13 Dobbs N, Burnaevskiy N, Chen D, Gonugunta VK, Alto NM, Yan N. STING activation by translocation from the ER is associated with infection and autoinflammatory disease. Cell Host Microbe. 2015;18(2):157-68.

14 Konno H, Konno K, Barber GN. Cyclic dinucleotides trigger ULK1 (ATG1) phosphorylation of STING to prevent sustained innate immune signaling. Cell. 2013;155(3):688-98.

15 Wang Q, Liu X, Cui Y, Tang Y, Chen W, Li S, et al. The E3 ubiquitin ligase AMFR and INSIG1 bridge the activation of TBK1 kinase by modifying the adaptor STING. Immunity. 2014;41(6):919-33.

16 Mukai K, Konno H, Akiba T, Uemura T, Waguri S, Kobayashi T, et al. Activation of STING requires palmitoylation at the Golgi. Nat Commun. 2016;7:11932.
17 Fang R, Jiang Q, Guan Y, Gao P, Zhang R, Zhao Z, et al. Golgi apparatus-synthesized sulfated glycosaminoglycans mediate polymerization and activation of the cGAMP sensor STING. Immunity. 2021;54(5):962-75.e8.

18 Gui X, Yang H, Li T, Tan X, Shi P, Li M, et al. Autophagy induction via STING trafficking is a primordial function of the cGAS pathway. Nature. 2019;567(7747):262-6.

19 Zhao B, Du F, Xu P, Shu C, Sankaran B, Bell SL, et al. A conserved PLPLRT/SD motif of STING mediates the recruitment and activation of TBK1. Nature. 2019;569(7758):71822.

20 Zhang C, Shang G, Gui X, Zhang X, Bai XC, Chen ZJ. Structural basis of STING binding with and phosphorylation by TBK1. Nature. 2019;567(7748):394-8.

21 Liu S, Cai X, Wu J, Cong Q, Chen X, Li T, et al. Phosphorylation of innate immune adaptor proteins MAVS, STING, and TRIF induces IRF3 activation. Science. 2015;347(6227): aaa2630.

22 Tanaka Y, Chen ZJ. STING specifies IRF3 phosphorylation by TBK1 in the cytosolic DNA signaling pathway. Sci Signal. 2012; 5(214):ra20.

23 Tan Y, Kagan JC. Innate immune signaling organelles display natural and programmable signaling flexibility. Cell. 2019;177(2):38498.e11.

24 Martin M, Hiroyasu A, Guzman RM, Roberts SA, Goodman AG. Analysis of drosophila STING reveals an evolutionarily conserved antimicrobial function. Cell Rep. 2018;23(12): 3537-50.e6.

25 Goto A, Okado K, Martins N, Cai H, Barbier $\mathrm{V}$, Lamiable O, et al. The kinase IKKbeta regulates a STING- and NF-kappaB-dependent antiviral response pathway in drosophila. Immunity. 2018;49(2):225-34.e4.

26 Margolis SR, Wilson SC, Vance RE. Evolutionary origins of cGAS-STING signaling. Trends Immunol. 2017;38(10):733-43.

27 Kranzusch PJ, Wilson SC, Lee AS, Berger JM, Doudna JA, Vance RE. Ancient origin of cGAS-STING reveals mechanism of universal 2',3' cGAMP signaling. Mol Cell. 2015;59(6): 891-903.

28 de Oliveira Mann CC, Orzalli MH, King DS, Kagan JC, Lee ASY, Kranzusch PJ. Modular architecture of the STING C-terminal tail allows interferon and NF-kappaB signaling adaptation. Cell Rep. 2019;27(4):1165-75.e5.
29 Qi N, Shi Y, Zhang R, Zhu W, Yuan B, Li X, et al. Multiple truncated isoforms of MAVS prevent its spontaneous aggregation in antiviral innate immune signalling. Nat Commun. 2017;8:15676.

30 Shi Y, Yuan B, Qi N, Zhu W, Su J, Li X, et al. An autoinhibitory mechanism modulates MAVS activity in antiviral innate immune response. Nat Commun. 2015;6:7811.

31 Shi Y, Yuan B, Zhu W, Zhang R, Li L, Hao X, et al. Ube2D3 and Ube2N are essential for RIG-I-mediated MAVS aggregation in antiviral innate immunity. Nat Commun. 2017;8: 15138.

32 Zhu W, Li J, Zhang R, Cai Y, Wang C, Qi S, et al. TRAF3IP3 mediates the recruitment of TRAF3 to MAVS for antiviral innate immunity. EMBO J. 2019;38(18):e102075.

33 Yum S, Li M, Fang Y, Chen ZJ. TBK1 recruitment to STING activates both IRF3 and NFkappaB that mediate immune defense against tumors and viral infections. Proc Natl Acad Sci U S A. 2021;118(14):e2100225118.

34 Jeremiah N, Neven B, Gentili M, Callebaut I, Maschalidi S, Stolzenberg MC, et al. Inherited STING-activating mutation underlies a familial inflammatory syndrome with lupuslike manifestations. J Clin Invest. 2014; 124(12):5516-20.

35 Cao Y, Jiang LP. The challenge of diagnosing SAVI: case studies. Pediatr Allergy Immunol Pulmonol. 2019;32(4):167-72.

36 Fremond ML, Hadchouel A, Berteloot L, Melki I, Bresson V, Barnabei L, et al. Overview of STING-associated vasculopathy with onset in infancy (SAVI) among 21 patients. J Allergy Clin Immunol Pract. 2021;9(2):803-18.e11.

37 Lin B, Torreggiani S, Kahle D, Rumsey DG, Wright BL, Montes-Cano MA, et al. Case report: novel SAVI-causing variants in STING1 expand the clinical disease spectrum and suggest a refined model of STING activation. Front Immunol. 2021;12:636225.

38 Ergun SL, Fernandez D, Weiss TM, Li L. STING polymer structure reveals mechanisms for activation, hyperactivation, and inhibition. Cell. 2019;178(2):290-301.e10.
Human STING Is Regulated by an Autoinhibitory Mechanism
J Innate Immun 2022;14:518-531

DOI: $10.1159 / 000521734$ 